\title{
Old Structure - Young Stars The Stellar Content of the Centre of the LMC Bar
}

\author{
P. Linde and A. Ardeberg \\ Lund Observatory, Box 43, SE-221 00 Lund, Sweden, \\ (\{peter,arne\}@astro.lu.se) \\ B. Gustafsson \\ Uppsala Astron. Observatory, Box 515, SE-751 20 Uppsala, Sweden \\ (bg@astro.lu.se)
}

\begin{abstract}
.
The history of star formation and chemical evolution are studied in the LMC Bar centre with the HST PC and WFC and uvby photometry. Using dedicated image processing, we secured high spatial resolution and photometric quality. We present colour magnitude diagrams (CMDs) from PC and WFC $y$ and $b$ data. The PC provides a CMD close to complete to $V=23.5$, the WFC contributes favourable statistics on brighter stars. We find a population of stars seemingly around or younger than 0.2 Gyr comprising around $30 \%$ of the total amount of stars. Star formation seems to have decreased 3-0.2 Gyr ago. Older populations are aged 3-9 Gyr. Stars older than 10 Gyr seem rare if not absent. Our CMD morphology and, especially, our uvby metallicity index give $[\mathrm{Me} / \mathrm{H}]$ close to -0.4 , with older stars more and younger stars less metal poor.
\end{abstract}

\section{Programme and Observations}

In a field in the centre of the LMC Bar, we study the stellar content, the history of star formation, metallicity of individual stars and chemical evolution. The highest emphasis is on stars at and around the turn off region in the HR diagram. We use the Hubble Space Telescope (HST) with its Planetary Camera (PC) and Wide Field Cameras (WFC) plus intermediate band uvby photometry. For our present discussion, we neglect effects of interstellar absorption.

The target field has high stellar density and image crowding. However, excellent observing material and application of elaborate image processing, including image dithering, give good spatial resolution, high completeness and favourable photometric accuracy. Resulting standard deviations in the $y, b$ and $v$ passband data are below $0.015 \mathrm{mag}$ down to the upper turn-off region and below $0.020 \mathrm{mag}$ down to the lower turn-off region both for the PC and WFC frames. The PC $y, b$ and $v$ data have standard deviations around 0.04 mag at $V=24$ (Linde et al. 1998). Also our $u$ data are of high quality, albeit somewhat less so than the $y, v$ and $b$ data. 


\section{Luminosity Function (LF)}

The $y b$ CMD is a powerful tool for population analysis and age determinations. However, for our field, its application is, mainly due to effects of image crowding, limited to stars brighter than $V=24$, or approximately $M_{V}=5.5$, or even somewhat brighter. The corresponding LF, while less sharp as an analytical tool than the CMD, can be used for stars considerably fainter. Emphasising this advantage for derivation of the LF, we developed a new method (Snel 1998), including stars individually unidentified but statistically measurable. The LF of the LMC Bar, for statistical purposes derived from WFC data, agrees well with the solar vicinity one (Ardeberg et al. 1998a), even in details. It is still rising at $V=26$, or $M_{V}=7.5$. In both respects, it resembles the LF of LMC structures outside the Bar (Ardeberg et al. 1985).

\section{Colour Magnitude Diagram (CMD)}

Both PC and WFC data were used in our CMD studies. The PC CMD gives better accuracy and resolution, while the three WFC CMDs provide superior statistics, especially for brighter stars, important for age determinations. The resulting four CMDs, shown in Fig. 1, are rather similar in terms of over-all morphology. They include a total of around 16000 stars, out of which 1700 are in the PC CMD. Various tests show that the PC data are close to complete down to $V=23.5$, or $M_{V}=5.0$. For the WFC data, the corresponding completeness limit is at least $V=22.5$.

Using a galactic LF of the zero-age main sequence (ZAMS) from open clusters (Taff 1974), our number statistics and ZAMS fitting, we conclude that the young stellar component contains around $30 \%$ of the Bar stars. As an alternative, we used the same LF and giant branch statistics to estimate the old population contribution. The result is very similar to that based on the LF and ZAMS fitting. Stellar population ages were derived from comparisons with isochrones (Bergbusch \& VandenBerg 1992). The young population seems around or less than $0.2 \mathrm{Gyr}$, while the older populations range in age between 4 and $9 \mathrm{Gyr}$. From around 3 to 0.2 Gyr ago, star formation seems reduced.

\section{Evolution of the LMC Bar}

For the general Bar population, several authors have recently proposed ages below 2 Gyr and even below 1 Gyr, as summarised by van den Bergh (1998). Our data show a fundamentally different picture, which is more consistent with data on mass density and mass distribution. We have used corresponding results from a field close to the LMC core but outside the Bar (Ardeberg et al. 1985) to check for the influence of projection of halo stars. The disk and the halo have, in the magnitude range discussed, together a stellar surface density close to a factor 8 below that of our target field. Further, in our field, the corresponding practical factor is decreased for two reasons. First, the disk contribution should be small if any. Second, part of the contribution from the halo should be screened away by the Bar. We conclude that projection effects from structures outside the Bar can hardly influence our results very much. 


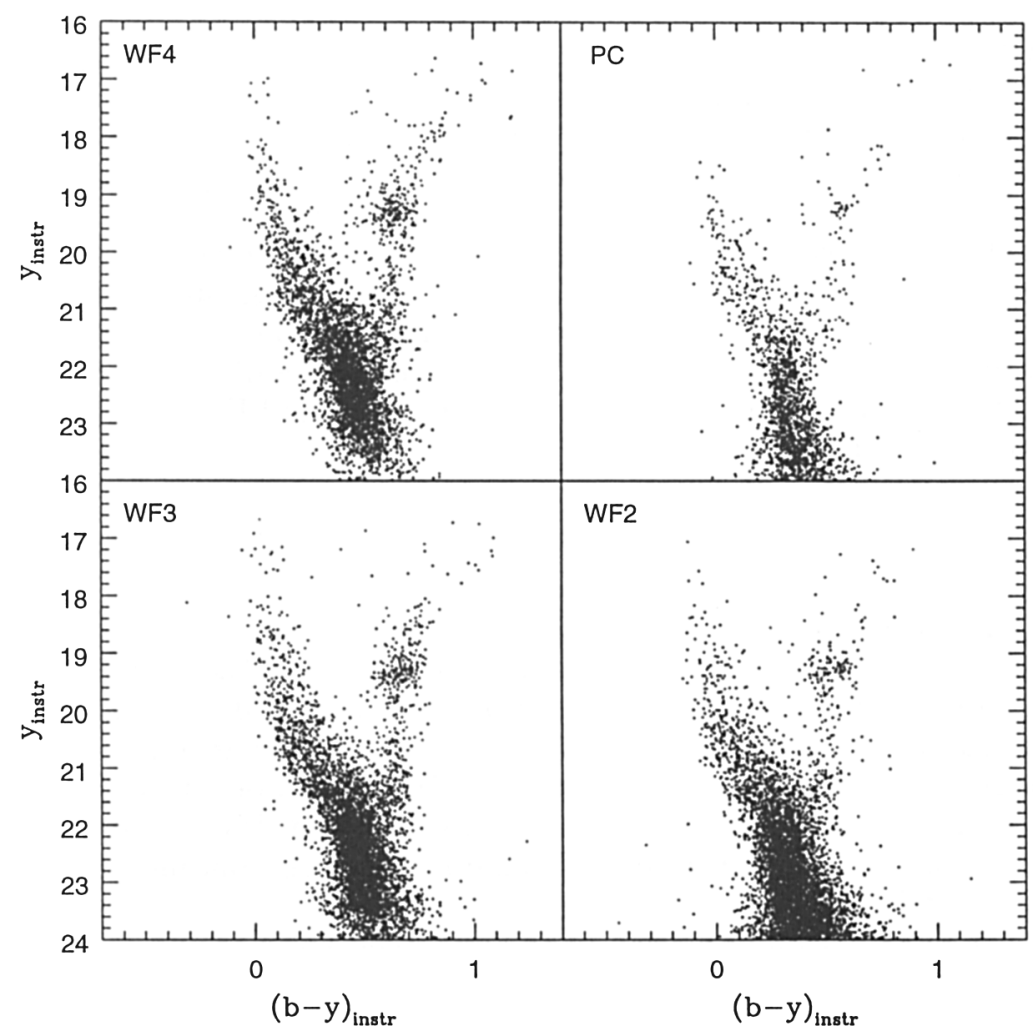

Figure 1. Colour-magnitude diagrams for the LMC core.

\section{5. $[\mathrm{Me} / \mathrm{H}]$}

Horizontal branch morphology and the colour of the horizontal and giant branches indicate an approximate average heavy element abundance corresponding to $[\mathrm{Me} / \mathrm{H}]=-0.5$. However, use of a combination of dedicated $m_{1}$ and $(b-y)$ indices in the uvby system gives more solid $[\mathrm{Me} / \mathrm{H}]$ data. From $\mathrm{PC}$ data for 292 turn-off stars with $19.5<y<22.0$ and relatively undisturbed images, we construct the $m_{1}$ vs. $(b-y)$ diagram in Fig. 2 to obtain individual $[\mathrm{Me} / \mathrm{H}]$ data (Edvardsson et al. 1998; Ardeberg et al. 1998b). We find an average [Me/H] of around -0.4. In Fig. 2, all turn off stars are plotted in the main diagram, while evolved stars brighter than $y=19.5$ are shown in an insert diagram in which clump stars are noted separately.

As can be seen from the $y, b$ and $v$ standard deviation data quoted above, the spread in Fig. 2 is much larger than that expected due to observational uncertainty only. Rather, it is a result of an extended age distribution. From a preliminary analysis, it seems clearly indicated that the oldest stars are considerably more metal poor and the youngest ones more metal rich than corresponding 


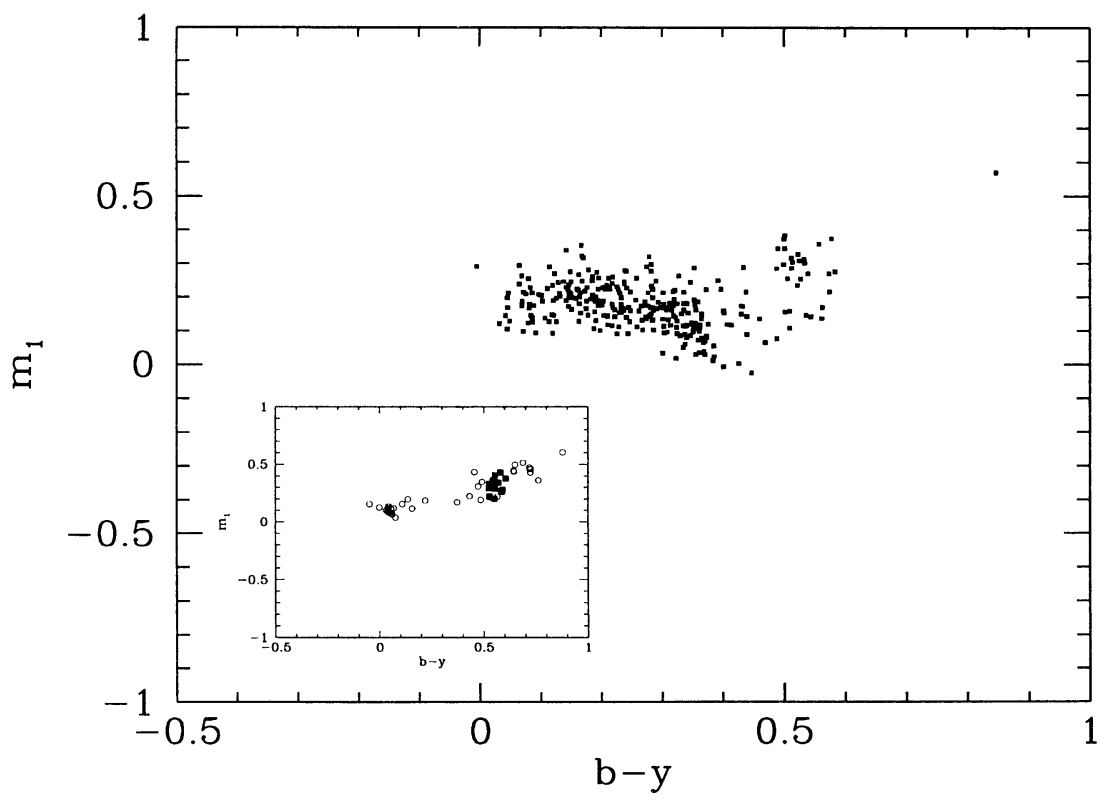

Figure 2. $m_{1}$ versus $(b-y)$ diagram for stars in the PC field with $19.5<y<22.0$. Insert: Stars with $y<19.5$, clump stars marked with filled squares.

to the average $[\mathrm{Me} / \mathrm{H}]=-0.4$. While our average $[\mathrm{Me} / \mathrm{H}]$ is close to that currently adopted for younger LMC stars, the variation with age has not been investigated before in the Bar. All such studies have, in practice, relied on clusters outside the Bar. For these clusters, the age distribution is dichotic (Geisler et al. 1997). We will return to the topic of evolution and heavy element abundance.

\section{References}

Ardeberg, A., Gustafsson, B., Linde, P. 1998a, Proc. IAU Symp. 190, in press Ardeberg, A., Linde, P., Lindgren, H., Lyngå, G. 1985, A\&A, 148, 263 Ardeberg, A., Lindgren, H., Lundström, I. 1998b, in manuscript Bergbusch, P.A., VandenBerg, D.A. 1992, ApJS, 81, 163

Edvardsson, B., Eriksson, K., Gustafsson, B., Joergensen, Plez, B. 1998, in prep. Geisler, D., Bica, E., Dottori, H., Clara, J.J., Piatti, J.F.C., Santos, J.F.C. 1997, AJ, 114, 1920

Linde, P., Ardeberg, A., Gustafsson, B. 1998, Proc. IAU Symp. 190, in press Snel, R.C. 1998, A\&AS, 129, 195

Taff, L.G. 1974, AJ, 79, 1280

van den Bergh, S. 1998, Proc. IAU Symp. 190, in press 